Conclusions In our study S. aureus strains obtained from children purulent-infectious diseases have high sensitivity to aminopenicillin with clavulan acid, meropenem, some cephalosporins, gentamycin. Among clinical strains of S. aureus we found methicillin- and even vancomycin-resistant microorganisms.

\section{1 \\ A RARE CASE OF LEMIERRE SYNDROME IN A 13 YEAR OLD CHILD}

doi:10.1136/archdischild-2012-302724.0881

II Velissariou, ${ }^{2} \mathrm{P}$ Papapavlou, ${ }^{2} \mathrm{H}$ Bairactaris, ${ }^{3}$ | Pierris, ${ }^{3} \mid$ Kolovos, ${ }^{1} \mathrm{M}$ Kalmanti. 'Pediatrics; ${ }^{2}$ Vascular Surgery; ${ }^{3}$ Radiology, Metropolitan Hospital, Athens, Greece

Object To present a rare case of Lemierre syndrome in a 13 year old girl.

Case Report The child presented with pyrexia, pharyngitis and dysphagia of 9 days duration. On the fifth day of pyrexia she developed a painful mass on the left side of her neck with bilateral tonsillar enlargement with exudate.

The patient underwent cervical ultrasound and magnetic resonance angiography of the brain that revealed septic thrombophlebitis of the left internal jugular vein. Antibody testing for cytomegalovirus, Toxoplasma gondii and Bartonella henselae were negative for active infection. Blood cultures did not have any growth. Computed tomography of the chest revealed multiple bilateral septic emboli, although the patient did not have any overt respiratory symptoms.

The patient received intravenous ceftriaxone and clindamycin for 3 weeks, followed by amoxicillin-clavoulanic acid orally for another 3 weeks along with anticoagulation therapy for 3 months in total.

Three months later, she was clinically asymptomatic, computed tomography of the chest was clear and the thrombophlebitis of the left internal jugular vein was stable.

Almost two years later, the patient remains in a very good clinical condition without any similar recurrences.

Conclusions Lemierre syndrome is a rare combination of tonsillitis and septic thrombophlebitis of the internal jugular vein caused primarily by Fusobacterium necrophorum, an obligate anaerobic gram-negative rod. In our case, we did not isolate the causative agent, however the patient had an excellent outcome with antibiotic and anticoagulation therapy without any surgical intervention.

\section{HEPATOSPLENIC CAT-SCRATCH DISEASE IN A 12-YEARS- OLD GIRL}

doi:10.1136/archdischild-2012-302724.0882

0 Diaz-Morales, JD Martinez-Pajares, JC Ramos-Diaz, J Trigo-Moreno, E FernandezGomez, M Miranda-Valdivieso. UGC Pediatria, Hospital Comarcal de Antequera, Antequera, Spain

Background Cat-scratch disease (CSD) is an infectious disease typically characterized by a self-limited regional lymphadenopathy. However, CSD can include hepatic and splenic involvement. There are few data in the literature regarding treatment of this situation, although administration of rifampicin associated with gentamicin and doxycycline or azithromycin is promoted.

Case Report A 12 year old girl was admitted because of 3 weeks sustained fever. No remarkable physical findings were presents. Laboratory findings in this admission only show CRP and ESR elevation and positive IgM for cytomegalovirus (CMV), together with fever defervescence, so she was discharged with probable diagnosis of acute CMV infection. However, she was readmitted a week later for recurrence of fever. Abdominal ultrasound detected multiples hyperechoic hepatic lesions and two larger splenic lesions, with no abnormalities of liver function. IgM (1/192) and IgG (1/3200) were positive for Bartonella henselae and PCR to CMV was negative, getting the diagnosis of an hepatosplenic form of CSD. Treatment with rifampicin and trimetroprim during 14 days was unsuccessful, therefore, triple therapy with rifampicin, doxycycline and azithromycin was started. Fever stopped after 6 days of treatment, but reemerged a week later, with a rebound of CRP and ESR levels. Finally, fever and analytical anomalies disappeared after several weeks, under monotherapy with azithromycin. No immunodefienciency was found.

Comments CSD must be suspected in the presence of prolonged fever with or without hepatosplenic involvement. In this case, little response was observed to the antibiotic therapy suggested in the literature, and evolution appeared to be self-limited.

\section{INCIDENCE OF LEISHMANIASIS IN A DISTRICT GENERAL HOSPITAL}

doi:10.1136/archdischild-2012-302724.0883

1,2S Damodaran, ${ }^{3} \mathrm{G}$ Erumbala, ${ }^{4} \mathrm{D}$ Abraham, 'S Nirmal. 'Paediatrics, James Paget Hospital, Great Yarmouth; 'PICU, Addenbrookes Hospital, Cambridge; ${ }^{3}$ Paediatrics, Milton Keynes General Hospital NHS Foundation Trust, Milton Keynes; ${ }^{4} \mathrm{NICU}$, Birmingham Children's Hospital, Birmingham, UK

Aim To study the incidence of Leishmaniasis in children under 5 years in a District General Hospital in the UK.

Method The microbiology records over 5-years (2005-2010) were reviewed. Inclusion criteria were age under 5 years and diagnosis of Leishmaniasis on bone marrow examination.

Results Two cases were identified.

Case-1: A 15 month-old-girl, family from east Timur, referred from primary care with weight-loss and a non-healing skin ulcer. She appeared undernourished with pallor, pyrexia and hepatosplenomegaly. FBC showed pancytopenia. Bone marrow examination confirmed Leishmaniasis. Her mother had intrapartum Leishmaniasis.

Case-2: A 22 month-old-boy presented with high fever and weight loss for 3 weeks. Examination confirmed pyrexia, pallor and hepatosplenomegaly. FBC revealed pancytopenia. Direct antigen test for Leishmania was positive. Leishmania Donovani complex was detected on bone marrow examination. There was no history of maternal infection.

Both children were born in United Kingdom with no history of foreign travel. They both responded well to treatment with ambisome.

Discussion Leishmania Donovani, the protozoan parasite, is transmitted in endemic areas by the insect vector Phlebotomine sandfly. In non-endemic areas, transmission is vertical. The infection can lead to impairment in cell mediated immunity and a 100\% mortality rate, if left untreated. Most cases of visceral Leishmaniasis occur in India, Bangladesh, Nepal, Brazil, and the Sudan. East Timor, although not endemic, has witnessed an upsurge of several confirmed cases recently. There was a history of maternal infection in only one of these two children. The source of infection in the other remains unidentified.

\section{THE VARIABILITY IN THE COURSE OF HEPATITIS B IN CHILDREN AFTER MOTHER-TO-CHILD TRANSMISSION AS A REASON TO ADJUST TREATMENT}

doi:10.1136/archdischild-2012-302724.0884

1.2B Kowalik-Mikołajewska, 1,2M Aniszewska, 1,2M Marczyńska. 'Department of Children's Infectious Diseases, Medical University of Warsaw; ${ }^{2}$ Hospital of Infectious Diseases, Warsaw, Poland

Background and Aims In Poland currently, despite obligatory hepatitis $B$ vaccination we expect the occurence of mother-to-child 